\title{
Germanica
}

\section{Gegenwartslyrik und Echtzeitkritik}

Martin A. Hainz: Poésie contemporaine et critique en temps réel

Martin A. Hainz: Contemporary Poetry and real-time criticism

\section{Martin A. Hainz}

\section{(Q) OpenEdition}

\section{Journals}

Édition électronique

URL : http://journals.openedition.org/germanica/7017

DOI : 10.4000/germanica.7017

ISSN : 2107-0784

Éditeur

Université de Lille

Édition imprimée

Date de publication : 26 juin 2019

Pagination : 149-164

ISBN : 978-2-913857-42-1

ISSN : 0984-2632

Référence électronique

Martin A. Hainz, "Gegenwartslyrik und Echtzeitkritik“, Germanica [Online], 64 | 2e trimestre 2019, Online erschienen am: 01 Januar 2021, abgerufen am 27 Februar 2021. URL: http://journals.openedition.org/ germanica/7017 ; DOI: https://doi.org/10.4000/germanica.7017 


\title{
Gegenwartslyrik und Echtzeitkritik
}

\author{
Martin A. Hainz \\ PH Burgenland/Universität Wien
}

\section{Denk- und Frageoptionen}

Gegenwartsliteratur und vielleicht vor allem -lyrik als deren knappste, beweglichste und mitunter konzentrierteste Ausprägung, mit Fokus auf dem Medium, durch das Literatur zu tun und zu sein vermag, was sie kann und ist, wirft Fragen auf - oder: Sie ist ein Fragen, die Frage nach dem, was sich ereigne, was sich durch sie ereigne. Neue Sprachmöglichkeiten sind neue Denk- und Frageoptionen. Dazu muss Lyrik forciert das werden, was potentiell alle Sprache ist, wie jedenfalls Werner Hamacher einmal behauptete: „Archiphilologie"1. Und das wiederum bedeutet, dass Literaturkritik, wenn es sie geben soll, Teil dieser Sprachbewegung sein muss: Gute Literatur ist Kritik der Literaturkritik.

Die Kritik muss in dem Sinne gegenwärtig sein, in dem es Literatur und Lyrik sind, nämlich: Gegenwart denkbar machend, während sie sonst eine notorische Vergangenheit meinte, die ahistorisch als unvergänglich gedacht wird, falls sie dann gedacht wird.

Gegenwartsliteratur ist also nicht jene, die gegenwärtig entsteht, sondern: für die Gegenwart, und durch (ihre) Gegenwart. Sie ist gegenwärtig in dem Sinne, dass sie etwas sieht oder erfindet, dessen es gegenwärtig vielleicht bedarf, wobei es nicht neu sein muss:

1. - Werner Hamacher, 95 Thesen zur Philologie, [Holderbank], Urs Engeler Editor, 2010, S. 1.

GERMANICA, 2019, LXIV, pp. 149 à 164. 
Die Irrelevanz der Frage, ob etwas neu ist, für die Frage seiner Gegenwärtigkeit hat Benjamin etwa an Klopstock angedeutet: Er schrieb, dass „manche Gedichte (Klopstocks) lauten, als seien es die heute gesuchten" 2 - und kann man sie so lesen, so sind sie es, genau da ist Gegenwartsliteratur gegenwärtig auch durch Gegenwarts- oder Echtzeitkritik, die wiederum die Gedichte vorwegnehmen. Sind nämlich diese nicht das, was als „Organ des [...] Aufwachens“ ${ }^{33}$ sich (gleichfalls mit Benjamin) beschreiben ließe?

\section{Gelegenheit, Gelegenheitsgedichte}

Literatur und Kritik sind darin einander verwandt: Es geht ihnen um das Wahrnehmen von Gelegenheiten, die nicht (auch: als erfindbare) zu finden zugespitzt einen Gegenwartsverlust bedeutete. Manchmal sind Literatur und Kritik miteinander identisch, immer wirken sie aber ineinander, zeitnahe sind sie, indem sie aufeinander sich beziehen, auf die Gegenwärtigkeit, die beide denken: Echtzeit meint hier nichts anderes. Der eine Aspekt ist die Gegenwart, die entsteht und wahrgenommen wird, und zwar durch das, was ,Gelegenheitsgedichte" ${ }^{\text {"4 }}$ sind, in der Art, wie Schmidt-Dengler ein Gutteil des Zeitgemäßen nannte; der andere ist, das zu sehen, was Sprache mitunter mehr denn der macht, der sie nicht gebraucht, sondern erprobend auf andere Intentions-Optionen ablauscht, also an seinem Geschriebenen oder aber dem anderer und auch den Beständen der Literaturgeschichte „,mit dem staubwedel“5 am Werk ist.

Keine Bedingung, aber ein Glücksfall ist dabei, jedenfalls möglicherweise, wenn die Interaktion dieser beiden Agenten des Sprachlichen dabei nicht durch Jahre oder - siehe Klopstock und Benjamin Jahrhunderte getrennt ist.

Man geht mit solcher Nähe gewiss auch ein Risiko ein, und zwar nicht nur jenes, dass vor allem eine kleinere Literaturszene Kritik mit Kumpanei verwechselt, sondern dass Echtzeit eben doch nur ein Fetisch ist: etwa jener der Beschleunigung, die aber auch Folge des Marktes sein kann, mit dessen Fetischen. Den Markt erfreut vielleicht das erwartete Buch mehr als das, das an der Zeit ist, seine Kritik wird den Bestseller suchen, anstatt vielleicht dem Buch, das gegenwärtig

2. - Walter Benjamin, Gesammelte Schriften, hrsg. v. Rolf Tiedemann et al., Bd. II: Aufsätze. Essays. Vorträge. Frankfurt a.M., Suhrkamp, 1999, 2. Aufl., Bd. II.1, S. 243.

3. - Walter Benjamin, Gesammelte Schriften, hrsg. v. Rolf Tiedemann et al., Bd. V: Das Passagen-Werk, Frankfurt a.M., Suhrkamp, 1998, 2. Aufl., Bd. V.1, S. 59.

4. - Wendelin Schmidt-Dengler, „Aber damals auf silbernen Schienen der Horizont", in: Gerhard Melzer, Stefan Schwar (Hrsg.), Friederike Mayröcker, Graz, Wien, Literaturverlag Droschl, 1999, S. 206-208, hier S. 206.

5. - Ernst Jandl, werke in 6 bänden, hrsg. v. Klaus Siblewski, München, Luchterhand, 2016, Bd. III, S. 130. 
wäre, Leser zu bescheren. Und die Übereilung („Gefällt mir - Gefällt mir nicht mehr") als solche wird gleichfalls verleitet sein, Kriterien zu verwenden, die keiner Muße bedürfen und sich nicht aus der Lektüre ergeben, womit Echtzeit das Risiko beinhaltet, dass die ,ausführliche und fundierte" Besprechung „rar"6 wird und verschwindet: Gegenwart braucht manchmal mehr Zeit.

Das darf und muss man sagen, will man auch nicht ,medienignorant"7 sein, wie es in Jörg Drews Überlegungen zum Phänomen von vor über 20 Jahren luzide heißt. Heinz Steinert bemerkte an Peter Glotz' Elogen auf die beschleunigte Kultur ebenfalls pointiert, dass dieser sie wohl auch darum pauschal gutheiße, weil er mit ,vier Assistenten und drei Sekretärinnen“", auf die Glotz mit Stolz verweist - man beachte übrigens die Geschlechterverteilung - jene Beschleunigung ,aus zweiter Hand“8 moderiert erfahre.

Übereilung: Dagegen verwehren sich Texte des Gegenwärtigen denn auch; so nahm einst Paul Celan einem, der in seinem Gedichtband herumblätterte, das Buch weg: So lese man ihn/seine Gedichte nicht ${ }^{9}$. Das kann man als Teil dessen auslegen, was der fast priesterliche Habitus des heiligen Sängers war, ohne Frage ist im deutschsprachigen Raum Literatur (und vielleicht ganz besonders Lyrik) spätestens im 18. Jahrhundert eine Kunstreligion geworden oder zu einem Teil derselben aufgestiegen ${ }^{10}$-, aber unberechtigt ist die Reaktion darum nicht. Es ist eine Entgegnung auf die Automatisierung und Beschleunigung; zur Textproduktion via Computer assoziierte Rose Ausländer (offenbar in den 1950er oder Anfang der 1960er Jahre), so entstünden nach formalisierbarem Publikumsgeschmack aneinandergereihte „Zufallsmetaphern“11. Das Pendant solcher Zurückweisung oder wenigstens Skepsis ist die schöne und nur prima vista bösartige Serie, die Daniela Strigl und Karin S. Wozonig für Volltext seit der

6. - Michaela Reichert, „Literaturkritik und neue Medien“, Lichtungen, Nr. XXXVIII 150, 2017, S. 201-202, hier S. 201.

7. - Jörg Drews, ,Zwischen Traditionalismus und Internet. Zur Beschleunigung der Literaturkritik“, Merkur, Nr. 591/6·52, Juni 1998, S. 531-537, hier S. 532.

8. - Heinz Steinert, „Die beschleunigte Gesellschaftsdiagnose. Skeptische Anmerkungen zu Peter Glotz' Buch Die beschleunigte Gesellschaft [...]“, Wespennest, Nr. 121/2000, S. 6-14, hier S. 14.

9. - Cf. u.a. Helmut Böttiger, ,'Alle Dichter sind Juden’. Der Auftritt Paul Celans bei der Gruppe 47 im Mai 1952“, Deutschlandfunk Kultur, 21.5.2017 - http://www. deutschlandfunkkultur.de/literatur-feature-vom-21-5-2017-sendungsmanuskript-alspdf.media.afa78409d62ce194fe7c19b98ed1c9b2.pdf (Zugriff am 13.5.2018), S. 27.

10. - Cf. Bernd Auerochs, Die Entstehung der Kunstreligion, Göttingen, Vandenhoeck \& Ruprecht, 2006.

11. - Rose Ausländer, Gesammelte Werke in sieben Bänden und einem Nachtragsband mit dem Gesamtregister, hrsg. v. Helmut Braun, Bd. 2: Die Sichel mäht die Zeit zu Heu. Gedichte 1957-1965, Frankfurt a.M., Fischer, 1985, S. 209. 
Ausgabe 3/2017 kuratieren, Zu Recht vergessen - die besten schlechten Dichter aller Zeiten. Hier wird implizit die Frage gestellt, welchen unzureichenden Mechanismen Literaturkritik gehorche, indem man die Textqualitäten dessen, was dann Gegenwart werden könne, ex negativo benennt. Man könnte hier auch noch auf den Streich von Franz Josef Czernin und Ferdinand Schmatz eingehen, die mit dem Band Die Reisen - In achtzig Gedichten um die ganze Welt eine Art von Lyrik beim renommierten Residenz-Verlag unterbrachten, welche nur eines zu tun vermochte, doch dies nachdrücklich: diesen auflaufen zu lassen, ihm nämlich nachzuweisen, dass ihn nicht die Frage interessiert habe, was „das gute Gedicht vom schlechten“"12 unterscheide...

Gegenwart ist, mit einer Formulierung Czernins und Schmatz', in der Literatur(-kritik) als Performieren wider ,in sich geschlossenen Schwachsinn"13 gegeben - ob sich das in Minuten und Sekunden ausdrücken lässt?

\section{Responsives Design}

Gleichwohl ist eine Sache der Zeit, und damit komme ich zum Thema anders und nochmals oder erstmals, ob das Gespräch über Lyrik zustande kommt: nämlich zeitnah, ihr Entstehen beobachtend, wobei die Kritik auf sie und sie auf die Kritik reagiert.

Das kann wie gesagt eine Chance meinen, eine, die vielleicht Workshops bieten, wo nicht etwa das Handwerk gelehrt wird, sondern diskutiert, wie man sich zu dem Text, den man schreibt, verhalten kann, ob ihn bewahren, was übrigens, wenn ein Text ein poetischer act sein kann, siehe H.C. Artmanns Proklamation hierzu ${ }^{14}$, kein Qualitätsurteil sein muss, sowie, ob und wie an und mit ihm arbeiten. Diese Chance bieten manchmal auch Lesungen, zumal gemeinsame Lesungen oder Performances, in einem „Raum aus Übereinkünften" 15 , worin gegeben werden kann, was am Jazz Jacques Derrida im Gespräch mit Ornette Coleman formuliert - darin geht es um die Idee, dass Menschen miteinander ein Gespräch führen, wobei aber keine diese Führung übernimmt ${ }^{16}$, das Ereignis entsteht

12. - Franz Josef Czernin, Ferdinand Schmatz, Die Reise. In achtzig flachen Hunden in die ganz tiefe Grube, Linz, edition neue texte, 1987, S. 7.

13. - Ebd., S. 10.

14. - Cf. Hans Carl Artmann, Das poetische Werk, hrsg. v. Klaus Reichert u. Hans Carl Artmann, Berlin u.a., Rainer Verlag, Klaus G. Renner, 1993, Bd. X, S. 13 f.

15. - Franz Josef Czernin, „Ein öffentlicher Raum (der Alten Schmiede gewidmet)“, in: Kurt Neumann (Hrsg.), Lesungsbilder. Österreichische Schriftstellerinnen und Schriftsteller lesen vor, Wien, Picus Verlag, 1995, S. 17-21, hier S. 17.

16. - ,the idea [...] that two or three people can have a conversation [...] without trying to dominate or lead it“. Jacques Derrida, Ornette Coleman, „The Other's Language. Interview, 23 June 1997“, übers. Timothy S. Murphy, Genre: Forms of 
also in der Aussetzung der Literatur, und zwar: der Lektüre, die da beispielsweise durch andere Literatur bekundet werden könnte.

Diese Chance bieten aber auch Plattformen, worin die Textproduktion und die Lektüre relativ zeitnah aneinander anschließen, eine Interaktion, die mich interessierte, als ich das Experiment der von mir zeitweise redigierten Hamburger Literaturkritikplattform fixpoetry (fixpoetry.com) mitgestaltete, unter dem Titel express! je drei Personen - einen Dichter und zwei Kritiker - online ins Gespräch kommen zu lassen, bei einem responsiven Design, das auch den erfreulich zahlreichen Lesern ${ }^{17}$ Kommentare zu posten gestattete.

Dabei zeigt sich, was man schon zu Beginn wissen konnte: Ein Projekt ist das nicht. Wenn es etwas ist, dann die Chance für ein sich erst formulierendes Projekt - wie fixpoetry insgesamt, und das ist derzeit das (auch finanzielle) Problem dieser Plattform: Sie ist keines der „Projekte, die einen Anfang haben [,] und ein Ende“, man kann diese Plattform so nirgends zwecks Förderung einreichen.

Das kann man entweder „eine langfristige Perspektive“ nennen und die „Flexibilität und rasche Reaktionsfähigkeit" 18 preisen, aber vielleicht ist es eher eine Perspektiv- und in Bezug aufs Projektieren eine Sprachlosigkeit. Oder man gesteht, dass man Projekte durch besagte Struktur fördern will, dann wären die Sponsoren Meta-Förderer - der Umstand, dass es das nicht gibt, ist dann bedauerlich. Immerhin müsste man aber eben solche Projekte auch skizzieren, also: was man so und nur so ermöglichen könne und wolle. Der Fokus auf Formate wie express! wäre dabei ein Weg, statt isoliert die Flexibilität zu preisen oder von einem Textkorpus $\mathrm{zu}$ sprechen, als wäre hier ein bemerkenswertes Archiv im Entstehen, über das man aber nicht zu sagen weiß: wovon.

Hier jedenfalls kam das Dreiergespräch zustande, um das es gehen soll. Dieses Experiment und der Verlauf einer Runde, an der ich mit Literaturkritiker Kristoffer Cornils aktiv teilnahm - diskutiert wurde mit Max Czollek dessen Text A.H.A.S.V.E.R. ${ }^{19}$ - ist Gegenstand meiner Beobachtungen und Überlegungen, die dann beschreiben, wie

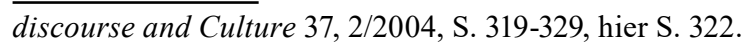

17. - Am Rande sei darauf verwiesen, dass das Wissen über Zugriffe samt Zugriffsdauer Rückschlüsse auf tatsächliche Lektüren ermöglicht, was ein Novum ist, gewöhnlich kennt man, wo nicht individuelle Zeugnisse oder Lektürespuren bestehen, nur ein Textkorpus und vielleicht bestimmte Zirkulationen, wenn es um die Rekonstruktion von Lektüre geht. Cf. auch René König, ,Geschichte und Sozialstruktur. Überlegungen bei Gelegenheit der Schriften von Rolf Engelsing zur Lesergeschichte“, IASL, 2/1997, S. 134-143.

18. - Julietta Fix, „SOS - saveoursite [Zwischenbericht]“, Fixpoetry, 28.02.2018 https://www.fixpoetry.com/feuilleton/notizen/2018-02-28/sos-save-our-site-zwischenbericht (Zugriff am 5.06.2018).

19. - Cf. Max Czollek, A.H.A.S.V.E.R., Berlin, Verlagshaus Berlin, 2016 (Edition Binaer, Bd. 1). 
Gegenwartslyrik, ihre diskreten Einheiten, Lyrikplattformen und eine solche Echtzeitkritik sich entwickeln, zueinander stehen und voneinander profitieren könnten.

\section{Max Czollek: A.H.A.S.V.E.R.}

Czolleks A.H.A.S.V.E.R. ist eine Studie der Eigenbewegung, der Herauslösung („Akt der Desintegration“20, schreibt die Jüdische Allgemeine fast prophetisch) und der Verantwortung, so könnte man es knapp umreißen. Max Czollek ist hier kurz vorzustellen, ebenso das Buch.

Max Czollek, 1987 in Berlin geboren, ist Lyriker, Essayist, Kurator und Mitglied des Autorenkollektivs G13. Unter anderem kreisen seine Texte um die Frage, inwiefern er Jude sei, was Jüdischsein für ihn bedeute. Dies ist keine Frage, die er ontologisch anginge, vielmehr fragt er, was einen gesellschaftlich als dem Judentum zugehörig konstituiere. Das ist zum einen der familiäre Hintergrund - übrigens ist er der Enkel des Widerstandskämpfers und Lektors Walter Czollek-, dann auch die Bildung, denn er besuchte die Jüdische Oberschule Berlin und legte hier auch 2006 das Abitur ab. Die Religion ist es in seinem Falle dagegen nicht, viel eher relevant ist, wie der Antisemitismus ihn zum Juden machte: Dieser und der (strukturell mitunter ähnliche) Philosemitismus haben Erwartungen, denen er sich ,desintegrativ“" entzieht, auch durch die Analyse der Vorurteile, über die er 2012 bis 2016 am Zentrum für Antisemitismusforschung der TU Berlin sowie am Birkbeck College in London promovierte.

Diese Erfahrung von Heteronomie ließ es sinnvoll erscheinen, mit dem Text A.H.A.S.V.E.R. nicht zum Gegenstand einer teils gründlich bundesdeutschen Kritik zu werden, sondern ins Gespräch mit ihr zu treten. Dann ist es aber auch der Text, der dazu einlädt. Dieser schildert, wie Josef oder Iosif sich „,durch Berge, Tannen, Nebel und Täler“ bewegt. Die romantische Landschaft aber trügt, sie ist bedrohlich, sie ist das Produkt eines Verdrängens und Verbergens; die Geschichte des ewig wandernden Juden ist eine der Rache an denen, die diese Geschichte erfanden oder verschuldeten: ,jüdische Selbstermächtigung“, wie Czollek es nennt. Und diese Heimsuchung wird nicht durch Festlegungen und eine Moral beigelegt, vielmehr bleibt immer die Frage, wer mit welchem Recht und von wem befragt spreche.

Schon der Band erschien mit einem Interview, das Micha Brumlik und Jo Frank mit Czollek führten, an diesen Übergang vom Text zur Antwort oder der Verantwortung knüpfte die Mischung aus Rezension und Gespräch an.

20. - Welf Grombacher, „Akt der Desintegration“, Jüdische Allgemeine, 21.07.2016 - www.juedische-allgemeine.de/article/view/id/26084 (Zugriff am 13.05.2018). 
Durch diese Eigenart des Textes bot es sich vielleicht besonders an, dieses dekonstruktive Langgedicht im Internet zu diskutieren: mit Blick auf die je nächsten Interpretamente, vielleicht aber auch das Weiterschreiben Czolleks, womit sowohl seine Antworten als seine Statements zu verstehen sind, vielleicht aber auch die Texte, die er hernach veröffentlichte und noch veröffentlichen wird, denn die Reaktion auf Verständnisse, manipulative Rezeptionsformen und Irrtümer ist wie angedeutet diesem Werk wesentlich. Aus heutiger Sicht ist A.H.A.S.V.E.R., soviel sei gesagt, wiewohl ich beim Interpretationsexperiment bleiben will, auch ein Präludium unter anderem des Vorhabens, das inzwischen mit dem Band Desintegriert euch! vorliegt.

\section{Czolleks A.H.A.S.V.E.R. als Reaktion auf Interpretationen}

Nicht wenige Rezensionen und Interpretationen unterließen diese Form der Einlassung, was man natürlich den Rezensenten nicht vorwerfen kann. Die Herangehensweise von fixpoetry war ungewöhnlich und ist nur durch die schon erwähnte Responsivität des Mediums überhaupt möglich, die denn auch manche Leser zunächst irritierte: Wann es denn losgehe, so wurde von einer Leserin gefragt, als der Polylog zunächst darum kreiste, wie man den Text denn anpacken könne.

Diese Haltung ist das Gegenteil dessen, was Czollek sonst erfuhr und bis heute mitunter erfährt. Denn die meisten Rezensenten vermeinen, schon ,alles“ $21 \mathrm{zu}$ wissen, was man wissen könne und müsse vom Text, aber auch vom Judentum. Dies ist zwar der Eindruck des Lyrikers, aber es ist kein unbegründeter Eindruck. Statt der Neugier besteht das Faszinosum der Alterität, die aber gar nicht bestehen darf, sondern aufs Exotische reduziert wird, vielleicht zudem auf das, was einem das gute Gefühl gibt, qua Solidarisierung auf der richtigen Seite zu stehen: als wäre das Jüdische genau eine Position, und zwar auch jenseits der nicht gewählten auf der Rampe oder in der Gaskammer. Dass man so eher in einer Beziehung zu dem steht, was die Gruppe konstituiert, die sonst keine ist, wird vergessen. Was nicht gewusst oder zur Kenntnis genommen wird, das ist das unhintergehbar Andere, das weder als exotisch fixiert werden darf noch vereinnahmt werden kann. Czollek schreibt, dass diese Rezeption den Juden etwa verpflichtet, „eine Entlastung oder Befähigung der Deutschen für deren eigenes Sprechen" 22 zu liefern.

21. - Max Czollek, Desintegriert Euch, München, Carl Hanser Verlag, 2018, S. 7.

22. — Ebd., S. 80. 
Damit bleibt das Schweigen derer, die der Nationalsozialismus zum Schweigen brachte und bringen wollte. Das, was zu sagen wäre und von Überlebenden und Nachkommen gesagt wird, wird bequem ersetzt, etwa durch kitschige Empathie-Exzesse wie denen Nora Gomringers, die über „Waggons“, die „Rampe“23 und „Au-schw-itz"24 redet, aus Sicht derer, deren Schweigen mehr vernehmlich macht, als es solch ein „Holocaust-Kitsch“ 25 zu tun vermag, wie Czollek beklagt. Nur noch obszön erscheint in der Tat die von ihm zitierte Laudatio auf Gomringer, als sie und insbesondere dieses Gedicht 2012 mit dem Joachim Ringelnatz-Preis bedacht wurde, das die Jury besonders hervorhob: Der Juryvorsitzende und Göttinger Literaturwissenschaftler Frank Möbus lobte, dass hier die ,reine Freude an der Kraft der Poesie“26 zuletzt bleibe; Freude an Kraft statt Kraft durch Freude, da kann man in der Tat nur gratulieren...

Dagegen ist nur mehr die Desintegration bis hin zu ,jüdische[n] Parallelgesellschaften“ $27 \mathrm{zu}$ formulieren - und der A.H.A.S.V.E.R.Text, auf den also zu kommen ist, hat das begonnen: als Verweigerung dem gegenüber, was als Vergangenheitsbewältigung das eher permaniert, was nicht zu bewältigen sein $\operatorname{mag}^{28}$. Es ist ein Text, der in seiner Unentschiedenheit verbleibt, wie Czollek auch sonst dort, wo die Betroffenheit ihren Anlass vergessen zu haben scheint, wieder auf diesen fokussiert - und aufs Unangemessene, das allen Texten eignet, die angemessen sein wollen, was man an Czollek als Humor (fast angemessen) wahrnehmen kann: „Darf man über Auschwitz lachen?“29.

Diese und ähnliche Versuche der Lyrik samt ihrer Rezeption scheitern eben unter anderem daran, dass hier etwas bewältigt werden soll, wovon die Überlebenden wie auch die Nachkommen der Opfer sehr verschiedene Ansichten haben, inklusive der Frage, ob sie denn zu dieser Gruppe gehören, wenn diese doch zunächst heteronom war, und ob diese Gruppe das zu bewältigen habe, was nur sie bewältigen könnte, falls diese Bewältigung nicht wie angedeutet unmöglich ist.

23. - Nora Gomringer, zit. nach ebd., S. 83.

24. - Ebd., S. 84.

25. - Ebd.

26. - Zit. nach ebd., S. 84, ein Bericht findet sich online, N.N., „AuschwitzGedicht 'Und es war ein Tag'. Dichterin Nora Gomringer bekommt Ringelnatz-Preis für Lyrik", https://sprengelhildesheimgoettingen.wordpress.com/2012/04/17/auschwitzgedicht-und-es-war-ein-tag/ (Zugriff am 24.02.2019).

27. - M. Czollek, Desintegriert Euch, a.a.O., S. 140.

28. - Cf. auch Martin A. Hainz, „Drehbuchkomplikation? - Lyrik nach Auschwitz", in: Bettina Bannasch, Hans-Joachim Hahn (Hrsg.), Darstellen, Vermitteln, Aneignen. Gegenwärtige Reflexionen des Holocaust, Göttingen, V\&R unipress, Vienna University Press, 2018, S. 81-97, hier S. 96 f.

29. - M. Czollek, Desintegriert Euch, a.a.O., S. 93; hier wäre auf das Werk des jüngst verstorbenen Edgar Hilsenrath mit Nachdruck hinzuweisen. 
Und was wäre eine Bewältigung? Wäre sie für alle gleich zu leisten, beinhaltete sie die Beschämung der Täter, die die Opfer schwerlich betrifft? „Die Täter können ihren Opfern nicht verzeihen“30, so fasste Friedrich Hacker diese gerne übersehene Seite in Worte.

\section{Der kritische Polylog}

Czolleks A.H.A.S.V.E.R. sollte darum anders diskutiert werden, wie dieser Text schon darauf reagiert, was an Vereinnahmung und damit Ausmerzung vorliegt. Er bewegt sich, er versetzt in Bewegung, mit dem Zitat Czolleks, ,,je schneller du läufst, desto schneller wechselt die landschaft", eröffnete ich darum das Gespräch.

Der Text verändert den, der spricht, sich aber, indem er sich selbst adressiert, genau darum nicht anzureden vermag, als Effekt des denominalisierenden Textes. Nun ist das eigentlich ein Moment der Rettung an dem, was ein Text ist - er hebt den Effekt auf, mit dem er begonnen hat, nämlich jenen des Benennens, wonach er ein Objekt hat bzw. hätte. „Der Tod ist das [...] Verschließen des Sinns. Er ist das Nomen.“31 Wenn aber der, der in diesem Text spricht, ein Täter ist - war, aber eben auch blieb -, dann ist der Text das, was ihn nun jagt. Er wüsste dagegen gerne (scheinbar: wieder), wer er ist, weil dann sein Unrecht, auch wenn ihn sein Name umbringt, doch keines wäre:

josef, iosif, joseph

wer bist du gewesen

wer bist du geworden? 32

Wenn dies die Frage ist, so sei es auch die an den Kritiker, der scheinbar und subjektiv schon alles weiß. Dagegen bietet der Text aber auch den Dialog an, worin er aber jedem ,den Blitz der möglichen Gewitter"33 abnötige und androhe, so damals wie heute meine These.

30. — Friedrich Hacker, zit. nach Wolfgang Müller-Funk, „Erinnerung als narrative Konstruktion des Vergangenen. Theoretische Überlegungen zu einer großen europäischen Erinnerung, der Shoah", in: Hajnalka Nagy, Werner Wintersteiner (Hrsg.), Erinnern-Erzählen - Europa. Das Gedächtnis der Literatur, Innsbruck, Wien, Bozen, Studien Verlag, 2015, S. 37-56, hier S. 45.

31. - Jean-Luc Nancy, „Entstehung zur Präsenz“, übers. Oliver Vogel, in: Christiaan L. Hart Nibbrig (Hrsg.), Was heißt „,Darstellen“?, Frankfurt a.M., Suhrkamp, 1994, S. 102-106, hier S. 104.

32. - Kristoffer Cornelis, Max Czollek, Martin A. Hainz, ,alles ist sichtbar, für den der sehen will““. Fixpoetry -www.fixpoetry.com/feuilleton/express/max-czollek/ edition-binaer-ahasver, 23.03.2016 (Zugriff am 22.04.2016); Zitate, die nicht gekennzeichnet sind, entstammen im Folgenden dem Gespräch.

33. - Michel Foucault, „Der Philosoph mit der Maske. Gespräch mit Christian Delacampagne“, übers. Birgit Wagner, in: Peter Engelmann (Hrsg.), Philosophien. Gespräche, Graz, Wien, Böhlau, 1985, S. 27-40, hier S. 32. 
Cornelis erwidert, dass es ,um einen stream, der die Richtung wechselt und dessen Verlauf von mehr als einem Faktor bestimmt wird“, geht. Das Problem aber wäre, dass das Gedicht diesen erfordere und doch nicht zulasse; „,Derweil Lyrik als smartphonekompatible und zeiteffiziente Literaturgattung gelobt wird, verträgt sich gebundene Rede nicht mit responsivem Design." Das hieße, dass Lyrik zwar rasch zu lesen sei (was vielleicht irrig ist), aber nicht antworte: weil ja gebunden - als gäbe nicht genau darum manches Gedicht seit vielen Generationen Fragen auf, neue Antworten indirekt zeitigend, weil die gefundene, offenbar durch die (oder eine) Zeit gültige Form das ermögliche.

Hier aber wird in genau dieses Fragen eingestiegen, in der Tat vollzieht Czolleks Text ja eine ,identitäre Prozessualität kritisch“ nach, so der erste Befund Cornelis', aber eben dies geschieht so, dass der Einspruch schon angeboten wird und geradezu immanent ist - sonst wäre der Zusatz, dass der Nachvollzug kritisch sei, auch sinnlos. Auch ist der Respons kein Reflex, die Hypothesen und Entgegnungen wurden darum auch nicht im vom Internet scheinbar geforderten Takt geliefert, vielmehr wurde jedenfalls meinerseits Echtzeit so aufgefasst, dass sie nicht der kulturellen Passform etwa des Internets angeglichen werden muss. Leider ist nicht immer die Zeit verzeichnet, die verstrichen ist, aber es lagen meist doch 1-2 Tage zwischen den Statements, nach einem gewissen Vorlauf selbstredend, um sich mit dem Ausgangstext zu befassen, es gab also Zeit genug, um (retrospektiv: im Einklang mit der Stavanger-Erklärung) die Texte auszudrucken und zu lesen.

Die Form der Präsentation ist also nicht reflexhaft - und gerade dadurch und durch den Formwillen responsiv, die gebundene Rede verbindet sich mit dem, was sie kritisiert, und ist die „Einladung [...], sich kritisch und intensiv [...] auseinanderzusetzen" 34 , und zwar hier mit dem, was sie referiert, wobei der Respons seinerseits ästhetisch sein könnte, immerhin ist dies die Substanz schon des Textes, über den wir auf fixpoetry sprachen. Czollek erwidert denn auch, dass Kritik als Reflexionsinstanz der Literatur mit dieser leide, zumal mit der marginalisierten Lyrik, die nun aber wieder ,davongalloppierend“ aufbreche, jedenfalls da, wo die Kritik sich und ihren Gegenstand falsch festlegte.

\section{Überforderung}

Bis zu diesem Punkt ist die Rezension also keine im üblichen Sinne, eher rezensiert wird, wenn überhaupt, das Rezensieren oder das Milieu, worin diesem Text - wie dem Juden - ein Platz zugewiesen wird Cornelis schreibt:

34. - Daniela Strigl, „Zentrales Aus für die Literatur?“, Die Presse · Paul Watzlawick-Ehrenring, Mi., 11.05.2016, S. V. 
Würde ich Max für A.H.A.S.V.E.R. mit Lob überschütten, wie weit wäre das von dem ,Versuch einer deutschen Gemeinschaft, sich von ihrer eigenen Täter*innengeschichte abzugrenzen" entfernt [...]? Würde ich ihn dafür hingegen kritisieren, weil ich Gewalt - egal, ob der Text sie eher als symptomatisch spiegelt als er dazu aufruft ablehne, wäre das nicht wiederum selbst ein gewalttätiger (Sprach-) Akt, der wiederum die Form eines strukturellen Antisemitismus [...] in sich trägt?

Es ginge stattdessen um eine Rezension, die das vermeidet, die sich überfordert ${ }^{35}$. Überfordert waren auch die Leser, zumal Julietta Fix, die die Webseite fixpoetry derzeit betreibt und für derlei Organisatorisches zuständig ist (bzw. gewesen wäre), nicht dafür gesorgt hatte, dass der nunmehr als e-Book erhältliche Text rechtzeitig auch so zu erwerben sein würde. Zumindest mir war das nicht klar, mit pdf auf dem Laptop und Buch auf dem Schreibtisch.

Allerdings war die Überforderung doch vor allem Unwille in Bezug auf Rede und Gegenrede: „Geht es hier um das Buch oder um die Kritiker?". Die Gegenwart wäre aber an der Gegenwartsliteratur genau dies, dass das sich nicht sagen lässt, aufgrund der Gegenwartsliteratur und der Gegenwartskritik.

Jener ,josef, iosif, joseph“ - nicht mit dem Autor zu verwechseln - ist ein Akteur, der doch nicht allein als auszurechnende Reaktion erfährt, was sich in seiner Aktion ihm zuträgt.

Es gehe also um die Dynamik des Lesens als Weiterschreiben, Weiterschreiben des Selbst, das liest, und dessen, was es liest. Das Selbst aber muss sich verbergen, sich vor seiner Entbergung wandeln, wenn es das kann, es ist sich als seiner nicht vergangenen Vergangenheit ausgesetzt. Alternativ müsste es vergehen - kann es?

[...] versuche, dem fadenkreuz zu entwischen schlage haken bei tag gib ruhe zur nacht verberge deine fackel, rekapituliere die route [...]

[...] hat dir einen graben geöffnet in den legst du dich nieder merkst nach wenigen stunden das sterben will dir nicht glücken [...]

35. - „Wer nicht überfordert ist, ist nicht einmal gefordert", schreibt Blumenberg: „Der Raum zwischen den Extremen ist leer.“ Hans Blumenberg, Begriffe in Geschichten, Frankfurt a.M., Suhrkamp, 1998, S. 204. 
Fahrpläne und Oden mag es brauchen, so mein Schluss, übrigens an Klopstocks Geburtstag - jedes Urteil verortet sich ja auch in seiner/ der Zeit als kultureller Passform. Cornelis überspringe ich hier, Czollek jedenfalls bestätigt, wenn er das als Autor so könnte, den Weg:

Vordergründig geht es um die Aneignung der antisemitischen Legende vom Ewigen Juden. [...] Mich interessiert(e) an diesem Text die Rückbesinnung auf den Bannspruch als literarische Form.

Der Täter wird zu einer Figur wie Ahasver, er sucht den Namen, mit dem er vielleicht dem Tod überantwortet würde, aber nicht der Schuld. Die Weise, wie das Opfer durch die Tätersprache hindurch noch redet, indem es diese falsch klingen lässt, ist die eine Seite: Der „Sprecher der Vergasten“ „lügt" 36 , schreibt beispielsweise Albert Drach in Unsentimentale Reise. Die andere Seite ist der Täter in der Opfersprache, die als Sprache nicht die des Opfers mehr ist, sondern etwas wie Rache oder Strafe denkbar macht.

\section{„Inglorious Basterds“}

In Czolleks A.H.A.S.V.E.R. ist also der Täter seinem Text ausgesetzt. Das Medium rächt sich, das missbraucht worden ist, hier also die Sprache, die nicht Listen meint, nicht Deportationen, nicht den industrialisierten Massenmord. Sie verstößt den, der spricht, aus sich, weil er so gesprochen hat, überantwortet ihn dem Nomen bzw. also dem Tod oder der ewigen Schuld. Es ist die Logik des Czollek wichtigen Films Inglorious Basterds ${ }^{37}$, wobei die Wendung, es bedürfe der Inglorious Poets, von Adi Keissar stammt, die ein Buch dieses Titels veröffentlichte, aus dem Max Czollek übersetzte. Worin besteht diese Logik?

In Tarantinos Inglorious Basterds, woran sich der Inglorious Poet Max Czollek anlehnt, sucht die Jüdin (Shosanna Dreyfus/Emmanuelle Mimieux) durch das Medium, das sie zum Objekt machen soll, die heim, die sich für Arier halten, macht ihre Ausschließung zu deren Einschließung.

„Die Sprache als Tätersprache und der Film als Täterfilm [...] werden zur Anklage“, so mein Schluss; die Medien lassen die, die der Nazi als Opfer bestimmte, wie Czollek schreibt, sagen: „Hier sind

36. - Albert Drach, Unsentimentale Reise. Ein Bericht, München, Deutscher Taschenbuch Verlag, 1990, S. 332.

37. - US/DE 2009, D: Quentin Tarantino m. Eli Roth; cf. insbesondere die Kinoszene, online unter https://www.youtube.com/watch?v=yQncV8eYOBI (Zugriff am 12.05.2016). 
die Inglorious Basterds. Wir haben den Krieg gewonnen. So einfach kommen die Deutschen nicht davon." 38

Der Täter ist aufgrund dessen der, der nicht nur nicht souverän über das Medium verfügt - der Täter fällt vielmehr seinem Medium anheim, in ihm ist er als der bleibend kenntlich, „der kristall in kammern wuchtet / elephantenmasken trägt /auf stille wartet" - kenntlich also als ein Mörder, der nun aber diese Stille nicht bekommt.

Dieser erwarteten Stille kann er nicht entgehen. Für Czollek ist „Josef [...] eine Täterfigur“: ein Mensch, ,dessen Flucht nicht in erster Linie als [...] Verweis auf die Prozesshaftigkeit des Textes oder des darin angesprochenen Subjektes gedacht ist", wiewohl auch das gilt, sondern vor allem einer, der „eine sehr konkrete Verfolgung/ Verfluchung einer Täterfigur durch den Text" erfährt, was freilich, so würde ich es ergänzen, doch auf die Prozesshaftigkeit des Textes verweist, der ein Prozess ist und dem Täter den Prozess macht.

\section{Josif}

Das Langgedicht ist da vielleicht auch ein monologisches Drama. Es spricht aus dem Täter gegen den Täter - und in der Rezension besser keiner über das Gedicht, das sich ja jene holt, die souverän zu sein vermeinten. Der Täter wird im Text vor die Wahl gestellt, zu sterben oder stigmatisiert Zeuge gegen sich zu bleiben, Czollek assoziiert Tarantinos Bear Jew, vor dem die Täterfigur nun kniet, vor die Wahl gestellt, mit dem Baseballschläger niedergestreckt zu werden oder zu kooperieren, aber mit der Hakenkreuznarbe weiterzuleben.

Man könnte auch den Kinobrand, den Shoshana organisiert, diskutieren: worin nämlich die, die sterben, zuvor ihre Projektion sehen, die ihnen das sagt, was sie sind: Sie sind Mörder und sterben, wobei das verheerende Feuer wie ein Reflex auf den Allesbrand (holokaustos) wirkt ${ }^{39}$.

Der Text urteilt, er urteilt über die Urteile und die, die sie fällen. Er urteilt, indem er diese Urteile vorträgt, er als Medium urteilt anders, flottiert, das Urteil wirkt, indem der Täter/ Schreiber sich so oder anders schuldig (ge)macht (hat).

\section{Vorwissen und Solidarität}

Der Leser weiß immer Falsches und/oder zu wenig; und er sagt meist zu Eindeutiges. Czollek rekurriert auf das Format des Dialogs pragmatisch auch mit einem Seitenhieb auf jene richtende Kritik, die

38. - M. Czollek, Desintegriert Euch, a.a.O., S. 172.

39. - Cf. auch Drehli Robnik, „Geschichte im Gesicht. Cinephilie ohne Rettung und Naziphobie ohne Vorbehalte in Inglourious Basterds", kolik, Sonderheft 12/2009: film, Okt. 2009, S. 11-17. 
sich dem Prozess noch entziehen will, wo ihr Verständnis schon als Problem kenntlich sein müsste:

Als problematisch stellt sich [...] die Überschreitung eines mehr oder weniger geteilten Referenz- und Wissenshorizontes deutscher Kritiker*innen heraus. Eine Rezensentin der FAZ führte diese Ambivalenz jüngst dazu, dem Autor ihr Unwissen zum Vorwurf zu machen.

Natürlich ist dieser Vorwurf nur als Frage des Lesers an sich formulierbar: „Wieviel also muß man wissen?“40 Der Text stellt die Frage nach dem zu Wissenden und Gelesenen oder zu Lesenden; das Tasten der Leser im Gespräch entspricht dem hier.

Der Text fragt den Leser, der Leser fragt sich. Und der Autor fragt ihn in diesem Polylog auch - notfalls durch Hinweise, die aber im Falle Czolleks und der FAZ solche sind, dass man sich nicht Antworten erwarten dürfe, dass Kompetenz den Text nicht ersetzt, weder eine mitgebrachte noch eine, die man mit ihm erwarb. Gegenwartsliteratur ist da durch die fast simultane Qualität nicht ermöglicht, aber wohl gefördert. Stattdessen ginge es um eine Kritik, die sich durch Solidarität mit dem Text auszeichnet, die mit ihm ,gemeinsame Sache [...] macht"“41, wie Hamacher schreibt. Literatur und ihre Kritik ergeben etwas Neues, wie Lektüre generell ins Schreiben münden kann, innerhalb eines Werks man denke an immer neu bearbeitete Lebensprojekte wie Klopstocks Messias, Goethes Faust oder noch Musils Mann ohne Eigenschaften -, aber auch intertextuell. Gericht hält dabei der an den anderen Text anschließende Text immer auch und meist vor allem über sich selbst. Zeitnähe kann hier entstehen, nicht in der Fetischisierung der Nähe, wie auch die Verklärung der Zeitferne schaden kann, die sonst paradox Zeitnähe zu werden vermag, sie wieder intensiver herzustellen geeignet.

Das beste Ergebnis ist vielleicht immer eine Gegenwärtigkeit: die Intelligenz, die diese herstellt und aus ihr spricht, aus Text wie Deutung nämlich; und hier sei (fast zeitfern) die Fortsetzung von Derridas Reflexion zum Jazz zitiert: Dessen Idee eines nicht von einem geführten Gesprächs verlange Intelligenz, in der Improvisation gestalten die Teilnehmer ein Puzzle, worin die Instrumente den Ton bestimmen ${ }^{42}$.

40. - Hans-Georg Gadamer, Wer bin Ich und wer bist Du? Ein Kommentar zu Paul Celans Gedichtfolge „Atemkristall“, Frankfurt a.M., Suhrkamp, 1995, 3. Aufl., S. 126.

41. - Werner Hamacher, Für - Die Philologie, [Holderbank]: Urs Engeler Editor, 2009, S. 55.

42. - „[T]he idea is that two or three people can have a conversation with sounds, without trying to dominate or lead it. What I mean is that you have to be ... intelligent, I suppose that's the word. In improvised music I think the musicians are trying to reassemble an emotional or intellectual puzzle in which the instruments give the tone." J. Derrida, O. Coleman, „The Other’s Language“, a.a.O., S. 322. 
Literatur wie Kritik leistete dies in unserem Experiment bis zu einem gewissen Grad, es war ein Verstehen der Muster, die vorliegen und an denen man nun teilhat, indem man sie und sich auf die Stimmigkeit zu befragen und Fortsetzungsmöglichkeiten zu diskutieren unternimmt: Das ist Echtzeitkritik zur Gegenwartsliteratur. Dies war für mich auch der Anlass, die Regel zuletzt zu unterlaufen - nicht als der Kritiker und Redakteur ein Schlusswort zu schreiben, sondern mein Statement qua Kommentarfunktion quer zum Diskurs zu positionieren.

\section{Geburt des Lesers}

Literatur ist ihre Deutung - nicht. Benannt, auf den Nenner gebracht, verortet und gegebenenfalls kanonisiert, ist sie keine, nur unbenannt wäre sie eine. Literatur geschieht darum, und zwar ständig, der Fokus darauf macht das Experiment, das ich zu umreißen suchte, spannend und lässt dieses das, was Gegenwart von Gegenwartsliteratur ist, klarer werden.

Die Interpretation ist [...] beides [...]: eine Aneignung des Textes mit den Mitteln der Interpretation und eine Reflektion der eigenen gesellschaftlichen Position, die gewisse ästhetische und normative Rezeptionsraster in Autor*in wie Kritiker*in einschreibt. Mir scheint hier eine Erweiterung jenes Konzepts der totalen Souveränität der Leser*innen angelegt, wie sie nach Barthes' Tod des Autors populär geworden ist $[\ldots]^{643}$,

so beschließt der Dichter den Polylog - und der Prozess bleibt, das Drama, nämlich als „Aushalten jener moralischen, ästhetischen und intellektuellen Ambivalenz, die entsteht, wenn der Andere einem als Anderer entgegentritt." So bleibt der Text, so wird er erst Text, in meiner versteckten Conclusio formulierte ich, dass der ,Autor [...] in der Tat als Instanz zuletzt sterben“ muss, wie Czollek es andeutet, doch: „um dauerhaft ,umgehen` zu können, als Gespenst umgehen, Festlegungen umgehend, Umwege gehend.“

Nach dem Text ist vor dem Text.

43. — Vgl. zur Souveränität bzw. „Geburt des Lesers“ Roland Barthes, „Der Tod des Autors“, übers. Matias Martinez, in: Fotis Jannidis et al. (Hrsg.), Texte zur Theorie der Autorschaft, Stuttgart, Philipp Reclam jun., 2000, S. 185-193, hier S. 193. 
